\title{
Der israelische Oberste Gerichtshof und die Besatzung: Rechtsprechung im Konflikt1
}

Der Oberste Gerichtshof (OGH) in Israel nimmt in einer besonderen Zuständigkeit die Überprüfung militärischen Handelns in den besetzten Gebieten vor. In dieser Funktion ist er schwerlich mit anderen Gerichten vergleichbar: nicht mit nationalen Gerichten, weil deren Zuständigkeit bei extra-nationalen Konflikten regelmäßig nicht gegeben ist und weil sie jedenfalls nicht kritisch die "eigene” staatlich-militärische Rolle zu bewerten haben; auch internationale Gerichte sind anders kontexturalisiert. Ihre juridischen Wertungen finden zeitlich und örtlich auf anderem Grund statt. Die Sachverhalte spielen sich inmitten einer militärischen Auseinandersetzung ab, also zwischen einer Besatzung mit deren (militärischer) Verwaltung und einer besetzten Bevölkerung. Allein die Tatsache, dass die Doktrin der "Nationalen Sicherheit" insoweit einer gerichtlichen Überprüfung unterzogen wird, stellt einen Meilenstein dar. Umgekehrt ist der Zugang der besetzten Bevölkerung zu einem Gericht des Besatzers ein Paradoxon, das eher politisch als juristisch zu betrachten ist und deshalb hier nicht vertieft behandelt wird, jedenfalls aber bei der Analyse der zur Entscheidung vorgelegten Verfahren mitgedacht werden muss.

Der OGH geht mit Fällen um, die sich direkt aus dem Konflikt ergeben und demnach einem aufmerksamen öffentlichen und politischen nationalen und internationalen Umfeld ausgesetzt sind. Einerseits ist für die Betroffenen der Weg zum höchsten Gericht des Besatzers weit. Andererseits ist für den israelischen Staat die gerichtliche Überprüfung des eigenen Vorgehens keine Pflicht. Insofern ist die Ausgangsthese hier, dass die Beteiligung zivilgesellschaftlicher Akteure an den Verfahren, insbesondere Menschenrechtsorganisationen, eine entscheidende Rolle bei der Geltendmachung der Rechte der besetzten Bevölkerung spielt.

In einer kurzen Einführung werden das israelische Rechtssystem und der OGH vorgestellt, um anschließend anhand von vier Fällen darzustellen, welche besondere Rolle den Menschenrechtsorganisationen bei denjenigen Verfahren vor dem OGH zukommt, die Handlungen der israelischen Armee in den besetzten Gebieten zum Thema haben.

\section{Der OGH und das Rechtssystem Israels}

Um der Frage nachzugehen, ob und warum Nichtregierungsorganisationen (NROen) so eine bedeutende Rolle in diesen Verfahren spielen, soll zunächst ein Überblick über das israelische Rechtssystem gewährt werden. Grundsätzlich ist festzuhalten, dass sich das israelische Rechtssystem wegen der Übernahme des britischen (Mandats-)Regelungsapparats am Common-Law-System orientiert, was u.a. bedeutet, dass es keinen Verfassungstext gibt. Einzig sogenannte Grundgesetze oder „Basic Laws" wurden nach und nach verabschiedet, um die wich-

1 Der Beitrag gibt ausschließlich die persönliche Meinung des Autors wieder. 
tigsten verfassungsrechtlichen Bereiche $\mathrm{zu}$ regeln. ${ }^{2}$ Immer wieder hat es Entwürfe und Arbeitsgruppen gegeben, die einen einheitlichen Verfassungstext ausarbeiten sollten. ${ }^{3}$ Aber u.a. wegen der heterogenen politischen Landschaft hat dies bislang nicht zum Erfolg geführt. ${ }^{4}$ Auch auf dem Gebiet der Grundrechte wurden 1992 zwei „Basic Laws” verabschiedet. ${ }^{5}$

Zusammengefasst lässt sich die besondere Rolle des OGH in diesen Verfahren auf drei Elemente stützen: die mit den Verfahren betrauten Richter_innen, die von Gesetzes wegen weite Zuständigkeit des OGH und die fehlenden Zuständigkeitsrügen von Seiten des Staates in den ersten Verfahren bezüglich der besetzten Gebiete.

\section{Die Richter_innen}

Der OGH genießt als Institution grundsätzlich einen hohen Stellenwert bei der Bevölkerung und insbesondere auch bei der politischen Führung. Dies stärkt die Legitimität der Entscheidungen. 6 Die beiden prägenden Persönlichkeiten - „dominant judges" - der letzten Jahrzehnte waren Meir Shamgar und Aharon Barak. Shamgar war sieben Jahre lang Generalanwalt, bevor er 1975 an den OGH kam und diesem zwischen 1984 und 1995 vorsaß. Aharon Barak war zunächst Dekan der Juristischen Fakultät in Jerusalem, dann Nachfolger von Shamgar als Generalanwalt und seit 1978 Richter am OGH sowie sein Nachfolger als Präsident des OGH von 1995 bis September 2006. Er wurde von Dorit Beinish abgelöst, der ersten Frau an der Spitze des OGH. Die langen Richterperioden entsprechen nicht nur angelsächsischer Tradition, sondern auch der Rolle der Rechtsprechung im jüdischen Recht. ${ }^{8}$ Die besondere Rolle der einzelnen Richter_innen lässt sich z.B. am Ansehen von Aharon Barak in Israel und international darstellen. ${ }^{9}$ Richter Barak hat fünf von sechs der hier in vier Fallgruppen vorgestellten Entscheidungen verfasst.

\section{Die Aktivlegitimation der Menschenrechtsorganisationen}

Im Abschnitt 15 der „Basic Law: Justizsystem“ findet sich die Zuständigkeitsbeschreibung des OGH. Dazu gehören „Fälle, in denen das Gericht die Notwendigkeit feststellt, zum Wohle der Gerechtigkeit ein Rechtsmittel zuzulassen, das keiner anderen gerichtlichen Zuständigkeit unterfällt"10 (Abschitt 15 (c)). Weiterhin steht es dem Gericht zu, den Behörden und ausführenden Beamt_innen Anweisungen hinsichtlich der Ausübung und deren gesetzlich festgelegter Tätigkeiten zu erteilen (Abschnitt 15 (d) (2)). Diese Zuständigkeit hat dazu ge-

2 Vgl. http://www.knesset.gov.il/description/eng/eng_mimshal_yesod2.htm .

3 Zuletzt etwa http://www.cfisrael.org//home.html.

4 Vgl. M. Wolffsohn, Politik in Israel. Entwicklung und Struktur des politischen Systems, Wiesbaden 1997.

5 Seit dem Kol Ha'am-Fall (1955) hatte der OGH eine judicial bill of rights ausgearbeitet, vgl. D. Kretzmer, The New Basic Laws on Human Rights: A Mini-Revolution in Israeli Constitutional Law?, Netherlands Quarterly of Human Rights Vol. 14/2, 1996, S. 173 ff., ders., Human Dignity in Israeli Jurisprudence, The Concept of Human Dignity in: E. Klein/ders. (Hrsg.), Human Rights Discourse, The Hague, 2002, S. $161 \mathrm{ff}$.

6 Vgl. D. Kretzmer, The Occupation of Justice: The Supreme Court of Israel and the Occupied Territories, State Albany (University of New York Press) 2002, S. 12. Hierzu auch Y. Dotan, Do the "Haves" Still Come Out Ahead? Resource Inequalities in Ideological Courts: The Case of the Israeli High Court of Justice, Law \& Society Review 1999, S. 1059 ff.

7 Vgl. D. Kretzmer (Fn. 6), S. 10.

8 Vgl. A. Fischer-Lescano, Globalverfassung. Die Geltungsbegründung der Menschenrechte, Weilerswist 2005, S. $132 \mathrm{ff}$.

9 Vgl. R. Goldstone, The jurisprudencial legacy of Justice Aharon Barak, Harvard International Law Journal Online 48/54, 2007.

10 Wie im gesamten Text eigene freie Übersetzung ins Deutsche. 
führt, dass Maßnahmen und Handlungen des Militärs und der Militärverwaltung

in den besetzten Gebieten vor den OGH gelangt sind.

Die fehlende begriffliche Konkretisierung ermöglicht wiederum eine weite Auslegung der Aktivlegitimation und bildet den normativen Rahmen für eine wichtige Präsenz der NROs und advocacy groups. Die Verfahren vor dem OGH sind eher informell, sie können unmittelbar und knapp beendet werden. ${ }^{11}$ So hat der OGH eine hohe Fallbearbeitungsquote, die wiederum der umfassenden Zuständigkeit geschuldet ist. ${ }^{12}$

Eine besondere Klagebefugnis oder ein Rechtsschutzbedürfnis für die hier relevanten Klagen und Anträge sind nicht vorausgesetzt. Demnach sind es häufig NROen oder Knesset-Abgeordnete, die Klagen erheben.

Die Verteidigung von Seiten des Staates wird von Anwälten der Abteilung für den OGH beim Justizministerium übernommen. Die Richter_innen am OGH haben vollstes Vertrauen in die Untersuchungen dieser Ministerialabteilung, in der Zeugen und Beweismittel sondiert und in das Verfahren eingebracht werden. ${ }^{13}$ Dabei handelt es sich um ein knappes Dutzend Jurist_innen im Staatsdienst ohne unmittelbare politische Zugehörigkeit, die ständig mit den 14 Richter_innen am OGH zusammenarbeiten. Auch wenn laut Dotan selbst die Verfahrensvertreter palästinensischer Kläger_innen diesen Beamten Anerkennung zollen, ${ }^{14}$ sind derartige Funktionsüberschneidungen zwischen Gericht und Verteidigung nicht unproblematisch.

\section{Die Klagen bezüglich der besetzten Gebiete}

Nach und seit dem Sechs-Tage-Krieg $1967^{15}$ hat sich der OGH mittels der erwähnten Zuständigkeitsfiktion mit Klagen betroffener Palästinenser, KnessetAbgeordneter oder unterschiedlicher Gruppen, die Interessen der betroffenen Palästinenser vertreten und dargestellt haben, befasst. Dies sind etwa Physicians for Human Rights, Adalab Legal Centre for Arab Minority Rights in Israel, Association for Civil Rights in Israel, Gish a Legal Centre for Freedom of Movement, Btselem u.a.

Warum begann die inhaltliche Auseinandersetzung des OGH erst Ende der 1970er Jahre? Kretzmer weist auf mehrere Erklärungspfade hin. Einerseits kam eine neue Richtergeneration zum Zuge, die eine größtenteils im deutschen Recht ausgebildete und aus dem deutschsprachigen Raum stammende Richterschaft ablöste, deren juristischer Einfluss bis heute reicht. ${ }^{16}$ Die neue Generation wurde in Israel und im common-law-System ausgebildet. Weiterhin erwähnt Kretzmer das politisch-historische Zeitmoment, in dem die Überzeugung unantastbarer militärischer Überlegenheit durch den Jom-Kippur-Krieg beendet war (1973), die politische Bereitschaft zum Dialog wuchs (Camp David) und in der Unterzeichnung der Friedenserkärung mit Ägypten 1979 gipfelte, womit eine andere Ebene der Auseinandersetzung durch den Staat eingeläutet worden sei. ${ }^{17}$ Inso-

11 Vgl. Y. Dotan, Judicial Rhetoric, Government Lawyers, and Human Rights: The Case of the Israeli High Court of Justice during the Intifada, en Law \& Society Review Vol. 33, 1999, S. 339.

12 www.court.gov.il.

13 Grundsätzlich vertritt der "Generalanwalt" den Staat, diese Abteilung untersteht ihm allerdings und tritt für ihn als spezialisierte Vertreter vor dem OGH in Verteidigung der Regierung, der Ministerien oder der Militärführung auf. Nur lokale Behörden werden ggf. von eigenen Anwälten vertreten, vgl. Y. Dotan (Fn. 11), S. 336.

14 Vgl. Y. Dotan (Fn. 10), S. 340.

15 Für die Zeit zwischen 1948 bis 1967 vgl. G. Seidman, "Judicial Administrative Review in Times of Discontent: The Israel Supreme Court and the Palestinian Uprising“, Israel Affairs, 2005, S. $42 \mathrm{ff}$.

16 Vgl. Oz-Salzberger/Salzberger, Die geheimen deutschen Quellen am Israelischen Obersten Gerichtshof, KJ 1998, S. $289 \mathrm{ff}$.

17 Vgl. D. Kretzmer (Fn. 6), S. 120. 
fern unterstellt Kretzmer, dass die Legitimation der Aktionen und Maßnahmen in den besetzten Gebieten auch über die gerichtliche Überprüfung hergestellt werden sollte. Dazu kommt, dass mit der Verabschiedung der Zusatzprotokolle zu den Genfer Konventionen, in denen die Pflichten von Besatzern noch verstärkt (1. Zusatzprotokoll) und auf nicht-internationale Konflikte ausgeweitet wurden (2. Zusatzprotokoll), ein engerer normativer Rahmen auf internationaler Ebene vorgelegt wurde, mit dem sich Israel auseinandersetzen musste.

In jedem Fall hat sich in dieser Zeit die Klagemöglichkeit für die erwähnten Organisationen herausgebildet, mittels derer Handlungen des Militärs auf palästinensischem Gebiet gerichtlich überprüft werden. Wenn Art. 15 c) der Basic Law: The Judiciary also die Zuständigkeit in Fällen gewährt, bei denen es „deems it necessary to grant relief for the sake of justice“, so geht die Klagebefugnis über eine Verbandsklage hinaus. Auch mit einer Popularklage ist dies nur ansatzweise vergleichbar, denn hier kann zwar grundsätzlich jedermann Klage erheben, die Zulässigkeit liegt jedoch im Ermessen des OGH, „for the sake of justice“. Hierbei handelt es sich um eine Art doppelrelevante Tatsache, die bereits über die $\mathrm{Zu}$ lässigkeit entscheidet.

\section{Die Rechtsprechung des OGH}

Der israelisch-palästinensische Konflikt ist in vielerlei Hinsicht besonders und aufgeladen, was eine rechtliche Einordnung nicht gerade erleichtert. ${ }^{18}$ Bei der hier zu behandelnden Zuständigkeit leitet der OGH Verfahren als oberstes Gericht Israels ein und bewertet das Handeln des eigenen Militärs. Der israelische Staatsapparat wird wiederum von einem Großteil der palästinensischen Bevölkerung als Feind angesehen. Gerade in diesem Spannungsfeld ist die Vertretung der jeweiligen Opfer wegen der politischen Sensibilität und Problematik, in dem Land des Besatzers vor Gericht zu gehen, oftmals allein durch Knesset-Abgeordnete oder direkt durch Menschenrechtsorganisationen möglich.

Die grundsätzliche Zuständigkeit des OGH für Klagen von Besatzungsopfern gegen Maßnahmen der militärischen Verwaltung oder einzelner Soldaten in Gaza oder dem Westjordanland ist in dieser Form von den Vertretern des Staates nicht nachhaltig bestritten worden. ${ }^{19}$ Bereits 1972 hat Richter Landau erklärt, dass mangels Einwendungen durch die Vertreter des Staates die Zuständigkeit des OGH gegeben sei, ${ }^{20}$ was im Ja' amait Ascan-Fall (1982) ${ }^{21}$ schließlich auch explizit festgehalten wurde. Warum der Staat diese Zuständigkeit anfangs nicht gerügt hat, liegt laut Shamgar daran, dass eine gerichtliche Überprüfung stattfinden sollte, um Willkür zu verhindern und Rechtsstaatlichkeit auszuüben. ${ }^{22}$ Weniger idealistisch sind die Thesen Kretzmers, wonach eine Anerkennung der israelischen Institutionen durch die besetzte Bevölkerung stattfinden und eine politische Legitimation der Machtausübung in den besetzten Gebieten erreicht werden sollte. $^{23}$

18 Vgl. I. Canor, When Jus ad Bellum Meets Jus in Bello: The Occupier's Right of Self-Defence against Terrorism Stemming from Occupied Territories, Leiden Journal of International Law, 2006, S. $136 \mathrm{ff}$.

$19 \mathrm{Vgl}$. R. Shamir, 'Landmark Cases' and the Reproduction of Legitimacy: The Case of Israel's High Court of Justice, Law and Society Review Vol. 24 Nro. 3, 1990, p. 785.

20 Vgl. OGH, Israel Yearbook of Human Rights Vol. 5, 1975, S. 384.

21 Vgl. OGH 37 (4) PD 785.1982.

22 Vgl. M. Shamgar, Legal Concepts and Problems of the Israeli Military Government - The Initial Stage, Military Government in the Territories Administrated by Israel 1967-1980. The Legal Aspects, Jerusalem (Harry Sacher Institute for Legislative Research and Comparative Law), 1982. 
Anhand von vier Fällen (sechs Urteilen) soll gezeigt werden, welche Rolle zivil-

gesellschaftliche Akteure, insbesondere Menschenrechtsorganisationen in diesem Zusammenhang spielen können. Es ist zu beachten, dass es viele erfolglose Klagen vor dem OGH gibt, so dass die hiesige Darstellung zumeist erfolgreicher Klagen keine Repräsentativität aufweist, allerdings deutlicher die normativen und inneren Konflikte beleuchtet, denen die Richter_innen ausgesetzt sind.

\section{HCJ 4764/04: Rafab-Fall24}

In diesem Fall ging es um die zum Zeitpunkt der Entscheidung andauernden Kämpfe in Rafah im Gazastreifen, an der ägyptisch-palästinensisch-israelischen Grenze. Bei diesem Feldzug sollte das System unterirdischer Schmuggelwege zwischen Ägypten und Gaza zerstört werden. Das Militär hatte entsprechend der Rechtsprechung des OGH drei Maßnahmen ergriffen, um humanitären Problemen vorzubeugen und diese gegebenfalls auch zu lösen: a) Es wurde eine „humanitäre Telefonhotline“ eingerichtet, bei der Organisationen etwaige Verletzungen melden konnten, damit auf konkrete Vorfälle reagiert werden konnte, b) es wurde ein Koordinationsbüro eingerichtet, das in ständigem Kontakt mit dem palästinensischen Gesundheitsministerium, dem Roten Halbmond, dem Internationalen Roten Kreuz und Krankenhäusern vor Ort stand und c) in jeder Einheit wurde ein Verbindungsoffizier zu dem Koordinationsbüro benannt, der die angezeigten Verstöße überprüfen bzw. lösen konnte.

Die Kläger - Menschenrechtsorganisationen - wandten sich mit diversen Forderungen an das Gericht. Sie wollten die Rechtswidrigkeit eines tödlichen Beschusses einer Gruppe Zivilist_innen festgestellt wissen und forderten ferner, dass Krankenwagen auch ohne Genehmigung über die Hotline in die betroffenen Stadtteile gelassen werden sollten, dass medizinisches Material nach Rafah geliefert werden müsse, dass die Organisationen eigene Ärzte schicken und dass im Stadtteil Tel A-Sultan die Strom- und Wasserversorgung wiederhergestellt werden sowie Nahrungsmittel und Medizin in diesen Stadtteil geliefert werden dürften (vgl. Art. 20, 23, 55, 56, 63 Viertes Genfer Abkommen - IV GA).

Die Anhörungen haben nur einen Tag nach Eingang der Klage stattgefunden.

Der OGH unterstreicht, dass er (gerade bei einer andauernden Gefechtssituation) weder über militärisches Vorgehen ex ante entscheiden noch die Soldaten gefährden oder das militärische Ermessen bewerten kann, sondern einzig die rechtlichen Wirkungen militärischer Entscheidungen. Da hier im Rahmen der Klage keine unmittelbare Gefahr für die Soldaten bestand, ist die richterliche Überprüfbarkeit grundsätzlich gegeben.

Die Pflicht, zum Schutz der Zivilbevölkerung tätig zu werden, muss also im einzelnen Kontext und im Rahmen der Verhältnismäßigkeit und reasonableness bewertet werden (Abschnitt 23). Bezüglich der Ermöglichung einer Beerdigung für die Familien getöteter Zivilisten stellt der OGH etwa aus Art. 27 IV. GA eine solche Pflicht fest. Auch in Bezug auf die Strom- und Wasserversorgung setzt sich der OGH mit der Faktenlage auseinander und gibt dem Militär Verhaltensmaßnahmen auf. Dies ist nicht nur deshalb interessant, weil die Ereignisse im Rahmen eines andauernden militärischen Feldzuges stattfanden, sondern auch, weil das Militär parallel eigene Untersuchungen zu den Vorfällen angestrengt 
hatte. Trotzdem hat es sich der OGH nicht nehmen lassen, auf die völkerrechtlichen Grundsätze und Verpflichtungen hinzuweisen.

Einzig die Forderung einer der Klägerorganisationen, eigene Ärzte nach Gaza zu lassen, um die Situation und Bedürfnisse in den Krankenhäusern untersuchen zu können, lehnte der OGH nach dem Hinweis des Militärs ab, Ärzte aller anderen Nationalitäten dürften die Krankenhäuser sehen, aus Sicherheitsgründen aber keine Israelis. Bei der Abwägung zwischen den Sicherheitsinteressen/Schutzpflichten des Militärs und der Informationsbeschaffung der Organisationen urteilte das Gericht, dass es keine große Zumutung für eine Organisation sei, palästinensische oder ausländische Ärzte für die Besichtigung zu finden.

Die Rolle des OGH, der dem Militär während eines anhaltenden Feldzuges die Einhaltung humanitären Völkerrechts und die aktive Verpflichtung der Besatzungsmacht, für die Zivilbevölkerung zu sorgen, vorschreibt, ist etwa auch im Zusammenhang mit den Vorfällen der Bundeswehr in Afghanistan ${ }^{25}$ als eine bedeutende Instanz der Verhaltenskontrolle des Militärs zu sehen.

In diesem Urteil wird die Bedeutung der humanitären und Menschenrechtsorganisationen deutlich, die im Namen der betroffenen Zivilbevölkerung deren Rechtsverletzungen geltend machen können. Es handelte sich um bewaffnete Auseinandersetzungen, bei denen man nicht davon ausgehen konnte, dass die Betroffenen irgendeine Möglichkeit haben oder kennen, den OGH anzurufen. Insofern fungieren die Organisationen als eine Art privater Ombudsman und bringen ein Gerechtigkeitsverständnis „for the sake of justice“ zum Ausdruck.

\section{Die Grenzanlage/Mauer/Sperrzaun}

Wegen der Bedeutung dieser Fallgruppe werden drei Urteile mit unterschiedlichen Schwerpunkten vorgestellt, auch um die Entwicklung vor und nach dem IGH-Gutachten zum Bau der Mauer ${ }^{26}$ darzustellen. In dem Gutachten hatte der IGH u.a. festgestellt, dass Israel durch den Bau der Grenzanlage internationale Verpflichtungen verletze und demnach den Bau beenden, soweit es besetztes Gebiet betrifft, zurückbauen, Betroffene entschädigen und das Selbstbestimmungsrecht der Palästinenser gewähren müsse. ${ }^{27}$

\subsection{HCJ 2056/04: Beit Sourik-Fall28}

Wie in allen Fällen, die den Verlauf der Grenzanlage betreffen, ging es (erfolglos) um die Gültigkeit der Maßnahme als solcher und den konkreten Verlauf. Der Kommandeur der israelischen Besatzungstruppen im Westjordanland hatte die Inbesitznahme von Land verfügt, um die Grenzanlage an einer bestimmten Stelle zu errichten. Dem OGH lag die Frage vor, ob diese Maßnahmen rechtmäßig gewesen seien. Nach der zweiten Intifada Anfang der 2000er hatte die israelische Regierung verschiedene Aktionen eingeleitet, um die Gewalt unter Kontrolle zu bringen. Hierzu gehörte auch der Bau einer Grenzanlage, die in einzelnen Bereichen aus einer Betonmauer und zumeist aus einer Anlage mit Zäunen, Stacheldraht, Kontrollwegen u.a. besteht. Teile dieser Grenzanlage wurden auf be-

25 Vgl. Kaleck/Schüller/Steiger, Tarnen und Täuschen - die deutschen Strafverfolgungsbehörden und der Fall des Luftangriffs bei Kundus, KJ Heft 3/2010, S. 270 ff.

26 Zum IGH-Gutachten etwa K. Oellers-Frahm, Israel und die Mauer (IGH), Vereinte Nationen 3/2005, S. 104-107.

27 IGH, Legal Consequences ofthe Construction of a Wall in the Occupied Palestinian Territory, Gutachten, ICJ Rep. 2004, Abschnitte 149 ff., http://www.icj-cij.org/docket/files/131/1671.pdf.

28 Beit Sourik Village Council v. The Government of Israel et al., 30.6.2004. OGH, Judgements of the Israeli Supreme Court. Fighting Terrorism within the Law, 2005, S. 208 ff. = International Legal Materials Vol. 43, 2005, S. 1099. 
setztem staatlichem, andere auf privatem Grund und Boden errichtet. In den Fällen privaten Eigentums wurde - wie hier - die Inbesitznahme gegen Entschädigung und unter Verweis auf Sicherheitsaspekte zum Bau der Anlage verfügt: Das Verfahren der Inbesitznahme wird dem Ansprechpartner auf palästinensicher Seite übermittelt, der dies wiederum an die Betroffenen weiterleitet. Dann wird mit den Anliegern das Gebiet besichtigt. Binnen einer Woche haben die Betroffenen Zeit, Widerspruch bei dem israelischen Militärkommandeur einzulegen. Dabei wird möglichst eine Einigung erreicht, anderenfalls besteht eine weitere Wochenfrist, um vor dem OGH Klage zu erheben. Dies war hier der Fall.

Bürger der im palästinensischen Stadtrandgebiet von Jerusalem gelegenen Klägerin, der Gemeinde Beit Sourik, hatten derartige Inbesitznahmeanordnungen (order of seizure) erhalten. Die Einschränkungen, die durch den Bau entstanden und Zugang zu medizinischer Versorgung und Arbeit (Feldern, Olivenhainen, Ramallah ...) verwehren, verstießen aus Sicht der Klägerin gegen israelisches Verwaltungsrecht und humanitäres Völkerrecht. Dabei wurde argumentiert, dass diese Verfügungen a) widerrechtliche Enteignungsanordnungen in besetztem Gebiet darstellten, b) keine sicherheitsrelevante Begründung für den Bau an der vorgesehenen Stelle geliefert wurde und c) diese eine unverhältnismäßige Verletzung der Grundrechte der betroffenen Palästinenser zur Folge hätten.

Als eine Besonderheit in diesem Verfahren konnte die Klägerin ein Gutachten des Rates für Frieden und Sicherheit einbringen, welcher sich aus ehemaligen hochrangigen israelischen Militärs zusammensetzt und ursprünglich als eine der ersten Organisationen öffentlich für den Bau der Grenzanlagen plädiert hatte. In diesem Verfahren erklärte dieser Rat jedoch, dass der Verlauf des Zaunes an der konkreten Stelle keiner militärischen Notwendigkeit entspreche. Somit stellte sich eine „zivil“"gesellschaftliche Organisation von Militärs gegen die Entscheidung der aktuellen Heeresführung.

Der OGH bezieht sich in der Entscheidung auf seine ständige Rechtsprechung und stellt fest, dass Israel das Gebiet in kriegerischer Besatzung hält (occupatio bellica). Auch die Autorität des Militärkommandeurs folge demnach aus den völkerrechtlichen Vorschriften zur kriegerischen Besatzung, die sich hauptsächlich aus der Haager Landkriegsordnung (HLK) von 1907 ergeben und Völkergewohnheitsrecht darstellen. Auch das IV. GA gewährt diese Zuständigkeit. Der Militärkommandeur sei demnach an das Völkerrecht sowie die Grundsätze des israelischen Verwaltungsrechts gebunden, insofern auch an das Recht auf Anhörung, das Recht auf eine vernünftige Begründung und den Grundsatz der Verhältnismäßigkeit. Der OGH beschäftigte sich in diesem Fall mit zwei Fragen: Gibt es eine rechtliche Ermächtigung, dass der Militärkommandeur den Grenzzaun im Westjordanland bauen darf? Falls ja, wo genau darf er den Zaun bauen? Die Klägerin stützte sich hauptsächlich auf zwei Argumente. Erstens, dass ein Grenzzaun durch den Militärkommandeur nicht genehmigt werden darf, wenn seine Entscheidung, wie angeblich hier, aus politischen und nicht militärischen Gründen getroffen wird, und zweitens, dass eine Inbesitznahme privaten Grund und Bodens bei einer Besatzung verboten sei.

Das Gericht war jedoch der Ansicht, dass der Militärkommandeur die Entscheidung auf militärische (Sicherheits-)Argumente stützt und somit entsprechend dem humanitären Völkerrecht (Art. 23(g), 52 HLK und Art. 53 IV. GA) ermächtigt ist, wegen einer militärischen Notwendigkeit, das Land in Besitz zu nehmen. Tatsache sei aber weiterhin, so der OGH, dass der Militärkommandeur in besetzten Gebieten kein rechtsungebundener Souverän ist. Im Sinne des humanitären Völkerrechts sind ihm die Gewährleistung der Interessen und der Schutz 
der besetzten Bevölkerung auferlegt. Dies wird in Art. 46 HLK und Art. 27 IV. GA garantiert und vom OGH auch anerkannt. Im Spannungsfeld zwischen diesen Vorschriften und jenen, die eine Intervention ermöglichen, müsse demnach der Grundsatz der Verhältnismäßigkeit angewendet werden. In diesem Zusammenhang untersuchte der OGH, ob der konkrete Verlauf des Zaunes rechtmäßig war, und setzte sich ausführlich mit dem Widerspruch zwischen dem derzeitigen Militärkommandeur und den Ansichten des Rates für Frieden und Sicherheit auseinander, deren Vertreter den geplanten Verlauf für militärisch nicht notwendig hielten. Der OGH gewährt dem militärischen Ermessen jedoch in ständiger Rechtsprechung einen Wert, der nur mit gewichtigen Gegenbeweisen erschüttert, aber nicht durch gerichtliches Ermessen ersetzt werden kann.

Im Rahmen der Verhältnismäßigkeit im engeren Sinne wurde den Klägern insgesamt zugestanden, dass der durch den Verlauf der Grenzanlage entstehende Schaden der Bevölkerung unverhältnismäßig groß in Bezug zu dem Sicherheitsgewinn auf Seiten der israelischen Bevölkerung war. Zwar waren Tore vorgesehen, durch die palästinensische Bauern ihre Felder erreichen konnten. Der vorhersehbare Schaden durch Wartezeiten und Einschränkung der Bewegungsfreiheit sei jedoch nicht hinnehmbar.

Und so verpflichtete der OGH die Beklagte auf Änderungen im Verlauf des Grenzzauns.

Erwähnung finden in der Entscheidung auch die Aussagen mehrerer Bewohner des angrenzenden israelischen Dorfes Mevasseret Tzion, die ihre Angst um die guten Beziehungen mit dem palästinensischen Nachbardorf zum Ausdruck brachten und erklärten, bei dem geplanten Grenzverlauf sei dieses gute Verhältnis aus ihrer Sicht in Gefahr, und die Grenzanlage erfülle demnach gerade nicht das Ziel, die Sicherheit zu stärken. Die einzelnen Anordnungen wurden nach Abschnitten analysiert und der geplante Verlauf verworfen.

Zusammenfassend lässt sich also festhalten, dass das Gericht sich hier mit zwei besonderen Umständen auseinandersetzen musste: der militärischen Einschätzung hochdekorierter israelischer Veteranen, die sich gegen das ausgeübte militärische Ermessen wandten und in diesem Fall den sonst von Menschenrechtsorganisationen ausgeübten Erwartungsdruck aufbauten sowie den Aussagen der unmittelbaren israelischen Nachbarn, die eine Verschlechterung der Beziehungen zu den Bewohnern der palästinensischen Dörfer zum Ausdruck brachten. Diese Erwartungshaltungen von unterschiedlichen Warten der Gesellschaft aus, konnte das Gericht bestätigen, ohne das Militär vor den Kopf zu stoßen, indem die Rechtsprechung aufrecht erhalten wurde, militärische Entscheidungen nicht selbst zu bewerten, jedoch im Rahmen der Verhältnismäßigkeitsprüfung die „richtige“ Entscheidung wegen deren Wirkungen zu korrigieren. Hinzu kommt wohl auch, dass das Rechtsgutachten des IGH zum Bau einer Mauer kurz vor der Veröffentlichung stand.

\subsection{HCJ 7957/04: Mara'abe-Fall 29}

Dieses Urteil betrifft ein weiteres Stück der Grenzanlage und erging nach dem IGH-Gutachten. Alfei Menashe ist eine Siedlung, die ca. $4 \mathrm{~km}$ hinter der Green Line - der Grenze des Friedensabkommens von 1949 - liegt. Zum Schutz dieser

29 Zaharan Yunis Muhammad Mara'abe, Morad Ahmed Muhammad Ahmed, Muhammad Jamil Mas'ud Shuahani, Adnan Abd el Rahman Daud Udah, Abd el Rahim Ismail Daud Udah, Bassem Salah Abd el Rahman Udah, The Association for Civil Rights in Israel v. The Prime Minister of Israel et al., 15.9.2005. Auf der Internetseite des OGH abrufbar unter http://elyon1.court.gov.il/files_eng/04/570/079/ a14/04079570.a14.pdf . 
Siedlung wurde der Bau eines Zaunes angeordnet, wodurch auch fünf palästinensische Dörfer vom Zugang zum restlichen Westjordanland abgeschnitten wurden. Dadurch werden faktisch nicht nur die Siedlung, sondern auch die fünf Dörfer Israel einverleibt.

Die Kläger waren Bewohner_innen der palästinensischen Dörfer sowie eine israelische Bürgerrechtsorganisation, die während des Verfahrens noch die Vertretungsvollmacht der Bürgermeister der betroffenen Dörfer vorlegte. Das Gericht stellte sich die Frage, ob auch israelische Bürger_innen in besetztem Gebiet, also hier in der Siedlung Alfei Menashe, geschützt werden dürfen, obgleich sie nicht von Art. 4 IV. GA umfasst sind (und obwohl die Umsiedelung in besetzte Gebiete gem. Art. 49 bas. 6 IV. GA verboten ist). ${ }^{30}$ Laut OGH sei dies aus zwei Gründen richtig: Einerseits ermögliche Art. 43 HLK das. Dass die Siedlungen laut IGH-Gutachten möglicherweise selbst illegal seien, habe damit nichts zu tun. Hierzu werde der OGH keine Stellung nehmen. Daneben handele es sich um israelische Staatsbürger, so dass es Staatspflicht sei, diese auch zu schützen. Trotzdem müsse man diese zu den israelischen Staatsbürger_innen auf israelischem Staatsgebiet abgrenzen.

Obwohl die Klage knapp 18 Monate nach Fertigstellung der Grenzanlage in dem konkreten Abschnitt erhoben wurde, erklärte der OGH, dass keine Verwirkung eingetreten sei, weil das Ausmaß der Einschränkungen erst später deutlich wurde. Im Anschluss setzte sich der OGH seitenweise mit dem IGH-Gutachten auseinander und der dortigen Feststellung, durch den Bau der Grenzanlage verstoße Israel gegen humanitäres Völkerrecht und internationale Menschenrechte. Der OGH erklärt, dass dieses Gutachten wichtig ist, weil es von der höchsten völkerrechtlichen Instanz verfasst wurde (Abschnitt 56) - obwohl es nicht bindend ist oder gar res indicata darstellt.

Nach Ansicht des OGH - wobei auch einige Richter_innen des IGH diese teilen (Buergenthal, Higgins) - liegt der Unterschied im Ergebnis zwischen der Rechtsprechung des OGH und dem Gutachten des IGH in den Kenntnissen zur Faktenlage, da auch der IGH erklärt habe, dass kein völkerrechtlicher Verstoß vorliege, wenn die Rechtsverletzungen mit militärischer Notwendigkeit, nationaler Sicherheit oder öffentlicher Ordnung gerechtfertigt werden könnten. Hier hätten dem IGH aber gerade keinerlei Informationen zur Sicherheitsbedrohung vorgelegen.

Anders als im Beit Sourik-Fall kommt der OGH zu dem Schluss, dass trotz Verletzung der Verhältnismäßigkeit im engeren Sinne keine alternative Route möglich sei. "Without it, there is no security for the Israelis. With it, there is a severe injury to the fabric of life of the residents of the villages [...] It seems to us that the time has not yet come to confront this difficulty, and the time may never come" (Abschnitt 116). Vielmehr wurde den Beklagten aufgegeben, nach Möglichkeiten eine Alternativroute zu suchen.

In diesem Fall vertrat eine israelische Bürgerrechtsorganisation die fünf Bürgermeister der betroffenen Dörfer und übernahm somit den Einfluss auf das Ermessen des $\mathrm{OGH}$ hinsichtlich der doppelrelevanten Tatsache „for the sake of justice“, der OGH setzte sich ausführlich mit dem IGH-Gutachten auseinander und kam zu dem Schluss, dass er mangels alternativer Route nicht selbst über die grundsätzliche Notwendigkeit der Grenzanlage und damit der Durchsetzbarkeit der Wahrung der Verhältnismäßigkeit befinden wolle. 
Diese Klage wurde vom Bürgermeister des palästinensischen Dorfes Bil'in, Ahmed Issa Abdallah Yassin, erhoben. Es ging auch hier um die Inbesitznahme/ Einziehung von Land mittels militärischen Anordnungen, etwa Az. Tav/40/04 vom 24.11.2004, die vom Militärkommandeur ausgestellt wurde, damit eine Sperr- und Grenzanlage auf dem Gebiet des Dorfes Bil'in entstehen könne. Das Dorf befindet sich in unmittelbarer Nähe der wachsenden israelisch-jüdischen Siedlung Modi'in Ilit, also im Westjordanland bzw. in Judea und Samaria. Gegen die ursprüngliche Anordnung vom 25.4.2004 gingen die palästinensischen Behörden vor. Der geplante Verlauf wurde im Anschluss an das Urteil im Beit Sourik-Fall überprüft und am 24.11.2004 neu gefasst. Noch immer blockierte der geplante Verlauf jedoch z.B. den Zugang zu den Olivenhainen, insgesamt ca. die Hälfte der Dorffläche.

Das Besondere an diesem Fall ist jedoch, dass sich in Bil'in daraufhin friedlicher Widerstand formierte, ${ }^{32}$ an dem auch israelische und ausländische Staatsbürger teilnahmen, die sich jeden Donnerstag zu Demonstrationen trafen. Obwohl also in diesem Fall nicht eine Organisation, sondern die direkt Betroffenen die Klage erhoben, wurde durch die regelmäßigen Protestaktionen eine (zivilgesellschaftliche) Erwartungshaltung hinsichtlich der Gerechtigkeitsprüfung bei der Zulässigkeit zum Ausdruck gebracht.

Das Urteil, das am 4.9.2007 veröffentlicht wurde, untersuchte zwei grundsätzliche Fragen in diesem Zusammenhang: Entspricht die Handlung des Militärkommandeurs seiner Zuständigkeit und Legitimität? Und weiter, ist bei der Entscheidung Ermessen rechtmäßig ausgeübt worden? Hinsichtlich der ersten Frage vertritt der OGH die Auffassung, dass eine Inbesitznahme besetzten Gebietes aus militärischen Gründen entsprechend dem humanitären Völkerrecht gemäß Art. 52 HLKO und Art. 53 IV. GA möglich ist. Das Gericht geht jedoch nicht darauf ein, ob der Schutz der israelischen Siedlungen in den besetzten Gebieten eine auf diese Artikel gestützte Maßnahme begründen kann. ${ }^{33}$

Im Rahmen der zweiten Frage widmet sich der OGH dem Grundsatz der Verhältnismäßigkeit und wägt das militärische Sicherheitsinteresse mit den Menschenrechten der betroffenen Bevölkerung ab. ${ }^{34}$ Der OGH erklärt, dass der geplante Verlauf die Rechte der Bevölkerung unverhältnismäßig einschränkt und ordnet einen rechtmäßigen Verlauf an.

Hier ist hervorzuheben, dass der OGH erklärt, Bauvorhaben der Siedlungen dürften bei der Entscheidung über den Verlauf keine Rolle spielen, wenn diese noch nicht genehmigt worden sind oder noch nicht mit den Bauarbeiten begonnen wurde. Dies bedeutet, dass der OGH zwar nicht in die Entscheidungen des Militärs direkt eingreift, aber die Gründe der Entscheidung als unrechtmäßig darstellt. In dem Urteil wird weiter verfügt, dass die Bewohner von Bil'in zwischen 6 Uhr morgens und 20 Uhr ohne Genehmigung so lange die Tore der Sperranlage passieren dürfen, bis das Urteil umgesetzt ist. Der Erfolg in diesem einzelnen Verfahren beruht laut Aussagen der Vertreter der Klage auch auf dem durch die wöchentlichen Demonstrationen ausgelösten Druck. ${ }^{35}$

31 Yassin v. Government of Israel et al., 4.9.2007. Auf der Internetseite des OGH abrufbar unter http:// elyon1.court.gov.il/Files_ENG/05/140/084/n25/05084140.n25.pdf.

32 http://bilin-village.org/english/.

33 Vgl. D. Kretzmer (Fn. 6.).

34 Ob bei einer solchen Abwägung das Interesse aus Art. 52 HLKO und 53 IV. GA den gleichen Rang wie die Rechte der Betroffenen beanspruchen kann, ist fraglich. 
Tatsächlich ist das Leben in Bil'in weiterhin von willkürlichen Aktionen der Besatzungsarmee bestimmt. Mittlerweile ist auch ein Verfahren am Kanadischen Supreme Court gegen Unternehmen anhängig, die auf dem Gebiet von Bil'in entstehende Siedlungen bauen. ${ }^{36}$

\section{HCJ 3799/02: Early-Warning-Fall37}

Allein durch die Liste der Kläger wird auch in diesem Fall deutlich, welchen Wert die nahezu grenzenlose Aktivlegitimation für die gerichtliche Überprüfung der militärischen Aktionen in den besetzten Gebieten hat: Menschenrechtsorganisationen sammeln Informationen und wenden Ressourcen auf, um bei bestimmten Sachverhalten die gerichtliche Bewertung zu fordern.

In diesem Fall ging es um die militärische Taktik, bei Festnahmen in den besetzten Gebieten die Bevölkerung zu benutzen, um der Zielperson der Aktion seine bevorstehende Verhaftung mitzuteilen und dieser mittels eines Einheimischen ausrichten zu lassen, alle anderen (Unbeteiligten) aus ihrem Haus zu schicken, weil anderenfalls ihre Integrität nicht garantiert werde. In diesem Zusammenhang legten die Kläger auch Berichte vor, nach denen Personen aus der Zivilbevölkerung durch mutmaßlich mit Todesfallen versehene Häuser geschickt wurden oder gar als menschliche Schutzschilde für Soldaten oder Militärfahrzeuge verwendet wurden.

Im Verlauf des Klageverfahrens hatte das israelische Militär einen expliziten Marschbefehl ausgestellt, wonach solche Praktiken untersagt sind. Bezüglich des Frühwarnsystems besagt dieser, dass die Freiwilligkeit der Mitarbeit gesichert werden müsse und dass es nur darum gehe, die Risiken für die unbeteiligte $\mathrm{Zi-}$ vilbevölkerung zu minimieren.

Das Gericht stellte fest, dass die Verwendung von Zivilbevölkerung als menschliche Schutzschilde rechtswidrig sei, und prüfte im Anschluss das sogenannte Frühwarnsystem. In der Abwägung stehe einerseits der Schutz - sogar des Lebens - der bei der Zielperson befindlichen Menschen mittels dieser „Vorhut“ und, auf der anderen Seite, der völkerrechtlich verankerte Auftrag der Besatzungsarmee, die Zivilbevölkerung zu schützen und nicht durch derartige Aufträge gesteigerten Gefahren auszusetzen. Letztere ist laut OGH die gewichtigere Position (Abschnitt 24). Weiterhin müsse das Prinzip gewahrt sein, die Zivilbevölkerung von bewaffneten Auseinandersetzungen fern zu halten. Auch die freiwillige Zustimmung zu einer derartigen Unterstützung sei angesichts der Machtverteilung zwischen Besatzungsarmee und einzelner Person kein Argument. Schließlich könne in einer Kampfsituation nicht genau vorhergesagt werden, ob die Überlieferung der Warnung durch den Zivilisten diesen nicht doch in Gefahr brächte. Aus all diesen Gründen stellte der Vorsitzende Richter Barak fest, dass das sogenannte Frühwarnsystem gegen Völkerrecht verstoße.

36 Vgl. http://bilin-village.org/francais/xmedia/docs_divers/Bilin-Supreme-Court-Canada-20101006. pdf.

37 Adalah - The Legal Center for Arab Minority Rights in Israel, The Association for Civil Rights in Israel, Kanon - The Palestinian Organization for the Protection of Human and Environmental Rights, Physicians for Human Rights, B'tselem - The Israeli Information Center for Human Rights in the Occupied Territories, The Public Committee Against Torture in Israel, Center for the Defense of the Individual v. GOC Central Command Israel Defense Forces et al. (Early-Warning-Urteil), 6.10.2005. 
In diesem Fall ging es um gezielte außergerichtliche Tötungen (targeted killings) durch das israelische Militär von mutmaßlich als Terroristen identifizierten $\mathrm{Pa}$ lästinensern. Der OGH konzentrierte sich in seinen Ausführungen allerdings auf die Legalität jener Aktionen, bei denen die Zivilbevölkerung sogenannte Kollateralschäden erlitten hatte.

$\mathrm{Zu}$ der übergreifenden Fragestellung ist hier neben den Klägern aus der Zivilgesellschaft zu beachten, dass ein umfassendes Gutachten von Antonio Cassese vorgelegt wurde, das sich mit der Abgrenzung zwischen Kombattant und Zivilist befasste und feststellte, es gebe keine weitere Kategorie, etwa eines gesetzlosen Kombattanten (unlawful combattant). Aus dieser Interpretation folgt nicht, dass eine solche dritte Kategorie von Konfliktteilnehmern nicht rechtmäßig verfolgt und verurteilt werden könne. Aber aus der Haager Landkriegsordnung und dem humanitären Völkerrecht ergebe sich keine Möglichkeit, diese Terroristen „kriegsrechtlich legitimiert“ zu töten.

Einen früheren Parallelfall mitten in der zweiten Intifada hatte der OGH noch abgewiesen (HCJ 5872/01 vom 29.1.2002) und erklärt, er sei nicht in der Lage, die Mittel zu beurteilen, die zur Verhinderung mörderischer Attacken gewählt würden. Mittlerweile insistiert aber der OGH, dass neben der Anwendung von Besatzungsrecht immer wieder bewaffnete Konflikte in den besetzten Gebieten stattfinden, d.h. auch das ins in bello zur Anwendung komme. In dem Urteil selbst zitiert der OGH Cassese, der feststellt: „An armed conflict which takes place between an Occupying Power and rebel or insurgent groups - whether or not they are terrorist in character - in an occupied territory, amounts to an international armed conflict" (Abschnitt 18). ${ }^{39}$ Unter Hinzuziehung des IGH und des EGMR erklärt der OGH, dass bei Lücken (lacunae) des ins in bello als Teil des humanitären Völkerrechts auf das internationale Menschenrechtsrecht zurückzugreifen ist. Eine solche Lücke gebe es jedoch nicht, wenn das humanitäre Völkerrecht den Fall regelt. Zivilisten sind im Sinne des humanitären Völkerrechts gemäß \51(3) des Ersten Zusatzprotokolls zu den Genfer Abkommen (ZP I) in bewaffneten Auseinandersetzungen mit Militär ungeschützt, a) solange b) die Teilnahme an Auseinandersetzungen stattfindet und diese Teilnahme auch c) direkt ist. In Abschnitt 33 erklärt der OGH, dass ein Zivilist an einer bewaffneten Auseinandersetzung teilnimmt, wenn er Waffen verwendet, Sicherheitsinformationen sammelt oder sich auf Gefechte vorbereitet. Demnach ist es keine Voraussetzung, dass eine Waffe verwendet oder auch nur getragen wird. Zur Definition der direkten Teilnahme wird in Abschnitt 34 auf den gemeinsamen Art. 3 GK verwiesen, wo die aktive Teilnahme beschrieben wird. Diesen beiden Begriffen - direkt und aktiv - hat das Rwandatribunal (ICTR) die gleiche Bedeutung gegeben. ${ }^{40}$ Hier schließt sich der OGH an. Aus Sicht des OGH sollte diese Teilnahme nicht nur auf die Person begrenzt werden, die unmittelbar einen Angriff ausführt. Auch die ideellen Planer nehmen demnach direkt und aktiv an einer bewaffneten Auseinandersetzung teil (Abschnitt 37). Fraglich bleibt demnach nur noch, wie lange eine Teilnahme andauert. Hier stellt der OGH fest, dass eine strenge Beweislast für die andauernde Teilnahme bei der israelischen Armee liegt. Der OGH verweist auf internationale Gerichte, entscheidet jedoch nicht endand the Environment v. The Government of Israel et al. (11.12.2005/13.12.2006).

39 A. Cassese, International Law, 2005, Oxford University Press S. 420.

40 The Prosecutor v. Akayesu, ICTR-96-4-T, 1998. 
gültig über Rechtmäßigkeit oder Rechtswidrigkeit der gezielten Tötungen (Ab-

schnitt 60).

Auch in diesem Urteil haben Menschenrechtsorganisationen versucht, eine Art Ombudsman-Stellung einzunehmen und als rechtswidrig begutachtete Handlungen des israelischen Militärs einer gerichtlichen Überprüfung zuzuführen.

\section{Schlussfolgerungen}

Anhand dieser Fallbeispiele der Rechtsprechung des OGH lässt sich festhalten, dass Menschenrechtsorganisationen und beständige Forderungen der Zivilgesellschaft eine Auswirkung auf die Urteile haben, auch wenn dadurch das Leid der betroffenen Bevölkerung nicht unmittelbar gelindert wird. Die tatsächliche Anwendung der herrschenden akademischen Lehre in den einzelnen Urteilen ist eher die Ausnahme. Die Urteile des OGH bewegen sich auf einem schmalen Grat bei der Anwendung von (humanitärem und Menschenrechts-)Recht in der Wirklichkeit der Besatzung und bewaffneter Auseinandersetzungen. ${ }^{41}$ Auf diesem schmalen Grat kann die Artikulation von Menschenrechtsorganisationen und Zivilgesellschaft entscheidend sein. Diese betätigen sich wiederholt als eine Art Ombudsman und versuchen, gesellschaftliche Erwartungen zu artikulieren und somit Einfluss zu nehmen, konkret etwa auf das Ermessen des OGH hinsichtlich der doppelrelevanten Tatsache der betroffenen Gerechtigkeit - „for the sake of justice".

Auch wenn Kretzmer ${ }^{42}$ und Gross ${ }^{43}$ seit dem Beit Sourik-Fall einen Abschied von dem ursprünglichen Standpunkt des OGH feststellen, sich nicht mit Einschätzungen operativer Sicherheitsmaßnahmen des Militärs zu beschäftigen, so ist hierin kein neues Paradigma zu erkennen.

Dass Sicherheitsargumente eine gerichtliche Überprüfbarkeit nicht blockieren müssen, hat Richter Barak auf der nationalen Ebene schon frühzeitig festgestellt. ${ }^{44}$ Wegen der weit gefassten Aktivlegitimation und deren intensiver Nutzung durch zivilgesellschaftliche Organisationen, ist zu folgern, dass die Ausweitung der Rechtsprechung des OGH auf internationale oder transnationale Rechtsentwicklungen maßgeblich durch diese privaten Akteure erfolgt ist. Durch die Auseinandersetzung mit dem IGH-Gutachten, mit dem Zusammenspiel zwischen humanitärem Völkerrecht und Menschenrechtsrecht und hier insbesondere der Auseinandersetzung mit internationalen Gerichten ${ }^{45}$ lässt sich auch eine Durchbrechung des völkerrechtlichen Dualismus feststellen. Die Menschenrechte und das humanitäre Völkerrecht, die auf Grundsätze nationaler Sicherheit und militärisches Interesse stoßen, können eben auch von Individuen geltend gemacht werden, so dass es sich bei der Rechtsprechung des OGH um

41 Vgl. M. Klode, Máarab y Almonacid: ¿Dos casos de derechos humanos? Una propuesta de análisis jurídico-sociológico del Derecho internacional fragmentado, Revista Colombiana de Jurisprudencia Nr. 336, 2007.

42 Vgl. D. Kretzmer, Introduction, Israel Law Review, 2005. S. 8.

43 Vgl. A. Gross "The Construction of a Wall between The Hague and Jerusalem: The Enforcement and Limits of Humanitarian Law and the Structure of Occupation”, Leiden Journal of International Law Vol. 19 Nr. 2, 2006, Fn. 195.

44 OGH (1988), Israel Yearbook of Human Rights Nr. 33, 2003, S. 280 ff.

45 HCJ 769/02 vom 11.12.2005 zitiert u.a. Entscheidungen des ICTY, ICTR, den Interamerikanischen Gerichtshof, den Europäischen Gerichtshof für Menschenrechte, IGH, u.a.; zu Netzwerken im transnationalen Rechtsprozess und den gegenseitigen Beobachtungsverflechtungen siehe auch FischerLescano/Teubner, Regime-Kollisionen, 2006. 
den Ausdruck transnationaler Rechtsentwicklung handelt. ${ }^{46}$ Das bietet die Möglichkeit, selbst in einem der meist beachteten politischen Konflikte, die Geltung von Menschenrechten durch gerichtliche Überprüfung immer wieder einzufordern. 